Publicación semestral. ISSN 2215-4906

Volumen 81 - Número 1

Julio - Diciembre 2021

\title{
Contenedor de voces desde la narrativa propia frente a los discursos dominantes en la educación artística
}

\author{
Container of voices from the own narrative against \\ the dominant discourses in art education
}

María del Carmen Rossette Ramírez

DOI 10.15517/es.v81i1.47654

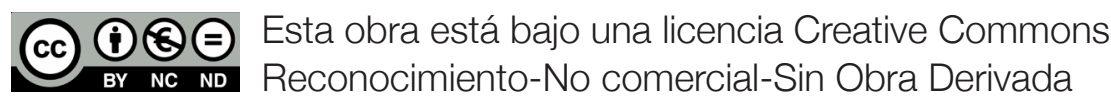


Reflexión

\section{Contenedor de voces desde la narrativa propia frente a los discursos dominantes en la educación artística}

Container of voices from the own narrative against the dominant discourses in art education

Ma. Del Carmen Rossette Ramírez1

Universidad Nacional Autónoma de México

Ciudad de México, México

Recibido: 12 de febrero de 2021 Aprobado: 22 de julio de de 2021

1 Profesora de tiempo completo en la Universidad Nacional Autónoma de México. Maestra en Artes visuales por la misma universidad. ORCID: 0000-0002-5458-5396. Correo electrónico: crossette@fad.unam.mx 
Contenedor de voces desde la narrativa propia frente

Reflexión

a los discursos dominantes en la educación artística

\section{Un acercamiento a las otras voces}

Las costumbres sobre la autoridad. La autoridad sobre las costumbres (Rodríguez, 1828, p. 88).

En esta cita, como en otros escritos, Simón Rodríguez critica las formas que establecen cómo nos conformamos de forma jerárquica ante la autoridad y en el mundo. Abre un perímetro alterno que ha generado lo que hoy conocemos como educación popular. A partir de este concepto, quiero enunciar desde donde escribo: lo hago en primera persona, ya que parto de una experiencia que sin duda es un punto de vista cimentado por mi trabajo como docente y tallerista. De esta manera, hago explícito el compromiso de compartir, de forma presente, el espacio del aula-taller, que se convierte en un lugar propicio para hacernos cargo de nuestra voz de forma colectiva y colaborativa; especialmente mi voz, que se encuentra entre lo académico, mediante la educación artística formal, y el taller de la eduación artística no formal. Utilizo un lenguaje coloquial como recurso pedagógico.

Asimismo, sigo la propuesta de Donna Haraway (2020), quien sugiere que el posicionamiento político surge de lo vivido mediante las experiencias, ya que estas no pueden ser neutrales, ni objetivas. En mi caso, enuncio como mujer, artista visual e investigadora, por eso lo presento y lo hablo en femenino. Teniendo lo anterior en cuenta, parto en este artículo con el planteamiento de hooks² (2003) sobre la investigación feminista: una no tiene que quedarse únicamente en el área académica, sino que debería abrirse a las demás personas. Esto me invita a una reflexión profunda sobre los discursos dominantes y los saberes que se manifiestan en el lugar de aprendizaje de cada una de las personas participantes del proceso educativo. En este espacio pueden abrirse posibles búsquedas de posicionamientos divergentes al discurso dominante:

Objectivity was made synonymous with an "unbiased stand-point." The professors who prided themselves on their capacity to be objective were most often those who were directly affirmed in their caste, class, or status position. Parker contends: "The oppression of cultural minorities by a white, middle-class, male version of 'truth' comes in part from the domi- neering mentality of objectivism. Once the objectivist has 'the facts,' no listening is required, no other points of view are needed.

2 bell hooks es una autora feminista quien por razones ideológicas y conceptuales, escribe su nombre con minúsculas.

ESCENA. Revista de las artes, 2021, Vol. 81, Núm. 1 (julio-diciembre), pp. 420-434 
The facts, after all, are the facts. All that remains is to bring others into conformity with objective "truth."' It is this will to bring others into conformity that merges with the will to dominate and control, what Parker calls 'the domineering mentality of objectivism.' Where there is domination there is no place for love. [La objetividad se convirtió en sinónimo de un "punto de vista imparcial". Los profesores que se enorgullecían de su capacidad para ser objetivos eran con mayor frecuencia los que se afirmaban directamente en su casta, clase o posición de estatus. Parker sostiene: "La opresión de las minorías culturales por una versión blanca, de clase media y masculina de la "verdad" proviene en parte de la mentalidad dominante del objetivismo. Una vez que el objetivista tiene "Ios hechos", no se requiere escuchar, no se necesitan otros puntos de vista. Los hechos, después de todo, son los hechos. Todo lo que queda es poner a los demás en conformidad con la "verdad' objetiva". Es esta voluntad de poner a los demás en conformidad la que se fusiona con la voluntad de dominar y controlar, lo que Parker llama 'la mentalidad dominante del objetivismo'. Donde hay dominación no hay lugar para el amor] ${ }^{3}$ (hooks, 2003, p. 128).

Así también lo manifiesta Dorotea A. Gómez Grijalva (2014), quien habla de un espacio dominado por una voz que se plantea como única denominada objetiva, por lo que aludo a mi experiencia y condición. Si bien no intento hacer una comparación entre dos sistemas de educación artística intento hacer evidente la complementariedad en la formación artística debido a que parto de mi trabajo en el aula y en el taller, y de la reflexión cuerpo territorio de lo que me implica estar presente en estos lugares como un proceso de investigación-acción, en los espacios educativos que iré demostrando con experiencias específicas, desde una postura crítica, anclada a mi contexto laboral.

En mi práctica educativa pretendo cultivar en los espacios de intercambio la comunicación desde lo dialógico, que se entremezcla con los múltiples eslabones de saber valiosos y no solo la comprensión occidental del mundo como única vía y fomentar la igualdad discursiva, en donde se manifiesta la urgencia de las múltiples razones que se producen como nuevas posibilidades (Corona Berkin, 2020). Romper con la objetividad y mediar entre los discursos dominantes y los saberes propios se vuelve indispensable en el ejercicio del aprendizaje horizontal (en donde se trabaja desde la equidad), para cambiar la estructura vertical (en donde se trabaja desde la jerarquía) que resulta violenta.

\footnotetext{
${ }^{3}$ Las traducciones al español son propias con propósitos de este texto.
} 
Es fundamental identificar las bases de los discursos dominantes establecidos en el desarrollo de la educación de las artes visuales y sus puntos de partida en la colonialidad del saber, del ser y del poder. En 1492, la modernidad nace como una empresa colonial que establece el reordenamiento del sistema mundial. Se condensa como un proyecto civilizatorio y, desde ahí, se puede vislumbrar la nueva formación de las academias de artes, las cuales acotan los oficios y especifican la formación artística. América Latina, desde hace más de 500 años, se convirtió en un experimento político, al ser un laboratorio geopolíico en el que se concretó la reconfiguración de un orden mundial, gracias al proceso de conquista-colonización que posibilitó un mundo moderno colonial, culminado con las políticas neoliberales. El resultado es un andamiaje de prácticas, formas y discursos que responden a un contexto contemporáneo que opera desde las políticas y los programas neoliberales, desde una lógica de privilegio dentro de un esquema global.

Al centrar y concentrar el conocimiento en todas sus posibilidades válidas, y según la perspectiva occidental, lo eurocéntrico privilegia su propia tradición, eliminando las otras formas de pensamiento con sus respectivas categorías estéticas. Esto hace imposible poder dialogar con diferentes culturas y promover la diversidad de pensamientos. La relación existente entre la modernidad y la colonialidad - que se contienen entre síhace que se entrecrucen temporal y espacialmente. Por tanto, el ejercicio de dominación se avala en cada trozo de tierra que fue saqueado y ordenado para los intereses de los que tienen el poder.

Bajo estas ya conocidas premisas, tenemos la necesidad de repensarlo todo, vernos frente a una alteridad y abrir la posibilidad de descentrar la mirada para pensar sobre nosotros de otra manera, de forma activa y participativa, en la costumbre de la exclusión de la reproducción de nuestra propia vida, la liberación, la lucha por construir nuestro lugar en el mundo, desde las narrativas propias, y desde nuestros saberes. Nos corresponde ir hacia el camino de la mezcla, de la traducción, de la construcción de nuevos lenguajes y de las exigencias globales, particularizando nuestro saber, ser y estar. Además, las implicaciones las traemos como introyectos al deconstruirnos desde una posición propia desde el aula o el taller.

El trabajo aquí propuesto tiene el interés de mostrar la relación existente entre educación artística formal y no formal y las posibilidades de un acercamiento a la emancipación de las inteligencias. Mi relación entre ambos sistemas de educación no se debe interpretar como un comentario sobre cada uno de ellos, más bien pretendo arrojar luz sobre la manera 
en que cada uno reflexiona sobre sus propias formas de articulación, las búsquedas de los procesos creativos que se tienen en la formación artística y sus replanteamientos. Desde el proceso de investigación-acción participativa en el aula-taller, en este trabajo se presenta un análisis de los discursos preponderantes y la posibilidad de aprenderlos para conocer y cuestionar las estructuras de poder.

\section{Mapa de ubicación: usted está aquí}

Podemos situarnos entre los vínculos existentes entre arte y educación y entre arte, conocimiento y procesos creativos. Todos ellos se encuentran en la intersección de experiencia y trabajo que se establece dentro del aula o taller. Me gusta pensar que existe la posibilidad de tender puentes en la vida misma, y no la abstracción que supone esa isla en la que se convierten los sistemas de adaptación social, que son las escuelas -sistemas de modelización- (Rolnik \& Guattari, 2006).

Aquí vale la pena reflexionar sobre qué carácter tiene el espacio que estamos construyendo. También hace falta revisar el papel de la docente o tallerista al convertirse en la persona que interviene entre quién dice que viene aprender y la institución. En el ejercicio de la educación artística existen tres participantes: la persona que está designada al frente del aula, la persona que decidió por cuenta propia estar en ese lugar y, por supuesto, el lugar en el que se lleva a cabo el acto pedagógico. Cada una de las entidades mencionadas tiene un peso específico en la operación. Podríamos decir que el aula y la persona designada comparten gran relación. Incluso, a veces sucede que la relación se hace estrecha; no obstante, tampoco se puede dejar de lado el libre albedrío de la persona que eligió estar en la institución.

Tomando la idea anterior, propongo argumentar que todas las personas somos una institución. ¿Cómo así? Las instituciones no se hacen solas, se van nutriendo de lo cotidiano, de las personas que vamos habitándolas y ejerciendo el espacio; lo convertimos en lugar y lo vamos colectivizando. Así, nos vamos apropiando de la institución. Esta inicialmente puede ser fría, con una enorme burocracia que lidiar. Nosotros la impregnamos de vida al compartir en comunidad.

Las instituciones en las cuales me gustaría compartir son de carácter público, entendidas como perteneciente al sistema educativo nacional, que se gestiona por la administración pública y es sostenida con los impuestos. Particularmente, la Universidad Nacional 
Autónoma de México (UNAM) es autónoma desde 1929 (Fundación UNAM, s.f.), mientras que la Fábrica de Artes y Oficios FARO Tláhuac es parte de la Red de FAROS que dependen de la Secretaría de Cultura del Gobierno de la Ciudad de México. La primera Fábrica de Artes y Oficios surgió hace veinte años en la periferia, en uno de los lugares con mayores índices de violencia y precarización de la Ciudad de México. Se origina con el propósito de acercar el arte y la cultura a las y los jóvenes en situación de riesgo. Cinco años después, se replicó el modelo en otros lugares de la ciudad. Cada una de estas Fábricas de Artes y Oficios (FARO) tiene su propia personalidad y su propia oferta para un público asiduo que busca ejercer y habitar el espacio.

Las dos instituciones en sus propias genealogías distan en sus objetivos particulares y en su misión. Sin embargo, las y los participantes de ambas hemos ido achicando esas distancias y hemos generado puentes entre ambas. Debido a lo anterior, la Fábrica de Artes y Oficios de Tláhuac, en sus ya casi 15 años de existencia, se ha llegado a convertir en un epicentro al sur-oriente de Ciudad de México. Su objetivo original fue generar una oferta de calidad de promoción cultural y formación en disciplinas artísticas y artesanales para el público juvenil. La apuesta era la descentralización de la oferta educativa y cultural del entonces Distrito Federal, ahora Ciudad de México. no obstante, en la práctica, fueron las familias, las infancias y las mujeres quienes ejercieron y dieron vida al espacio.

\section{La educación artística en tres patas}

Podemos identificar que existen otras maneras de hacer educación artística si partimos del postulado de Rancière (2011) sobre la emancipación de las inteligencias y la posibilidad de ver la ignorancia como una fuente de conocimiento en potencia y en acto, que está presente en la enseñanza junto con el aprendizaje. Eso refiere a un cambio en el paradigma, que rompe con el acto pedagógico normado, por lo que hace falta preguntarnos a qué nos referimos con acto pedagógico disruptivo. Esto puede entenderse como el acto de habitar el aula-taller y desmontar los roles establecidos de la autoridad que supone enseñar, lo cual posibilita a las y los estudiantes la disposición de aprendizaje-enseñanza.

A simple vista parece que se tiene en las escuelas una relación que responde a un orden patriarcal. Con esto, lo que intento generar es una serie de cuestionamientos que nos permitan hacernos de reflexiones propias en nuestro hacer, ya sea como parte del cuerpo de estudiantes, o bien, de la planta docente. ¿Para quién es útil el conocimiento? No es una pregunta novedosa. La encontramos en muchas(os) autoras(es) y postulados que buscan 
una resolución que nos ayude a tener sures globales más claros, porque es importante comprender las jerarquías sutiles que se hacen en el abordaje de llevar la clase. Es necesario ubicar la distancia que existe entre aprender y memorizar, ya que desde ahí los discursos dominantes operan y se reproducen, y los vemos naturalizado.

Como profesora, mi práctica educativa también la he puesto en cuestionamiento: me encuentro con la contingencia de intervención colectiva grupal en cada clase. El aula se convierte en una posibilidad de construcción común. De este modo, con un texto que leemos -entre todas las personas que conformamos la clase- y analizamos en conjunto, se hace un primer ejercicio de disrupción, pero esto es un primer paso que permite ubicarnos desde otras lógicas como concebirse desde la dignidad de la igualdad en las inteligencias y los saberes que ya traemos cada una de las personas. No recuerdo de manera clara cuándo fue mi primera ruptura ante el sistema hegemónico educativo al dar clases, pero sí tengo claro que rompí con las formas tradicionales y lo sigo haciendo. Estoy muy atenta a no caer en la tentación de la comodidad del escalafón al tener autoridad como profesora. Al buscar esta ruptura me di cuenta de que es necesario unir fuerzas con otras instituciones, personas y sobre todo con otras voluntades.

Una de esas complicidades la viví y la sigo teniendo con el grupo de investigación Mitote-Intervenciones Críticas desde el Arte Contemporáneo (ICDAC). Somos un puñado de docentes de la Facultad de Arte y Diseño de la Universidad Nacional Autónoma de México (FAD-UNAM) a quienes nos interesa romper las inercias y volver sinergias las opciones alternativas que rompen con las formas tradicionales y verticales de enseñanza dentro de la academia. Desde el 2014, colaboro con este grupo y en la actualidad contamos con el apoyo de un recurso que provee la UNAM a proyectos para innovar y mejorar la educación. Su objetivo es impulsar la superación y desarrollo del personal académico mediante el apoyo a proyectos que conduzcan a la innovación y al mejoramiento del proceso enseñanza-aprendizaje, y beneficien a las y los alumnas(os), tanto del bachillerato como de la licenciatura de la UNAM (Universidad Nacional Autónoma de México, s.f.). Este trabajo, por ejemplo, es realizado con el apoyo del Programa UNAM-DGAPA-PAPIME PE404520.

A través de estos ejes que he mencionado es que puedo observar como reproducimos en el aula los discursos dominantes que tenemos introyectos como un ejercicio naturalizado. Vale la pena recalcar que el sistema jerárquico aparece de múltiples maneras y se encuentra siempre en el salón de clase de manera velada. Cuando olvidamos por completo lo que aprendemos por nosotras mismas, dejamos de lado la educación basada en 
el mundo que nos rodea. Existe el peligro de que la cultura la hagan aquellos en el poder: los hombres. Los varones hacen las reglas y las leyes, las mujeres las transmiten (hooks, Anzaldúa, Sandoval, \& Brah, 2004). Gloria Anzaldúa habla de la tiranía cultural, donde se identifica qué tipo de hombre, no todos como humanidad, son los que poseen una clase social privilegiada, una raza y un lugar de poder que perpetúa el discurso hegemónico. Aquí cabe identificar cómo me relaciono con los discursos dominantes como individuo: desde una posición crítica, reconociendo a las y los estudiantes como agentes de cambio.

\section{La colonialidad en mi educación}

Sabemos que existe una relación entre el conocimiento, los saberes, la enseñanza y el aprendizaje. En la educación tradicional, la distancia que concurre entre la persona que educa y afirma el conocimiento con la escucha de manera pasiva me lleva a la pregunta: ¿qué conceptos relacionamos con la educación? Si me lo preguntan, pienso en estos:

- enseñanza / aprendizaje / instrucción / formación / didáctica / pedagogía / adiestramiento / institución / cátedra / precepto / estudio / educación / saber

¿Qué hacer con todas estas posibilidades? Si bien nos encontramos en todos estos conceptos la idea de que los perímetros pueden tocarse, también nos hablan de cosas que se parecen, pero que operan desde lugares distintos. Podemos decir que, al ser sinónimos, están en posición equidistante, por lo tanto, no se tocan. Una de las posibilidades en las que encuentro más potencial es la de continuidad que posee el aprendizaje. Walter Mignolo nos propone que en la educación "el desprendimiento implica el re-surgir, el re-emerger, el re-existir de culturas y memorias desprestigiadas en nombre de los ideales modernos y postmodernos" (Giuliano \& Berisso, 2014, pp. 62). Por lo tanto, hace falta la experiencia del aprendizaje para darnos cuenta de los saberes que ya poseemos e identificarlos como propios y como fuente de conocimiento.

Bien podríamos hacer una lista, como nos propone María Acaso (2016), de los lugares en los que aprendemos y en los que no: casa/aula/calle/libros/revistas/sitios web/fanzines/zines/reuniones/parque/plazas/fiestas/reuniones/tutoriales/canciones/poemas/cine/ biblioteca/bar. Todas(os) podemos seguir una lista interminable, de no solo lugares sino también de situaciones y personas. Así, entonces, podemos afirmar a través de nuestras acciones que el proceso de aprendizaje está siempre en construcción. 
Partiendo de lo anterior podemos hacernos la pregunta: ¿cuándo decimos que poseemos conocimiento? Parece que solo lo encontramos desde el logos que está planteado desde lo universal, como el pensamiento que se enmarca desde el a priori kantiano, como un juicio que aplica a todas las conciencias. Ahí podemos formular un cuestionamiento a esa teoría del conocimiento: ¿quiénes son esas conciencias?, ¿a quién le toca acotar desde ahí? Como respuesta indagativa, podemos decir que son formulaciones que responden a un orden occidental, como guía y único camino posible a transitar al conocimiento. Por eso comparto la idea de construir conocimiento situado, como dice Jabardo Velasco:

En la medida en que se construye desde la experiencia vivida y no bajo una posición teóricamente "objetiva", el conocimiento se crea dialógicamente. Frente al lenguaje objetivo y distante de otras formas de aproximación al conocimiento, en las epistemologías alternativas, la autora es central y está presente en el texto. En la epistemología feminista negra, la historia es contada y preservada en forma de narrativa y no desde una posición analítica (Jabardo Velasco, 2012, p. 34).

En lo cotidiano, nos hallamos con el acto lingüístico, que portamos en el cuerpo. En esas construcciones nos encontramos de manera expresa con la estructura de dominación de poder. No se ha ido: sigue y desafortunadamente seguirá. Por ello, es oportuno preguntarnos: ¿cómo se vive la colonialidad en el conocimiento?

Cuando leo la pregunta, si he vivido alguna forma de colonialidad del conocimiento, pienso en toda mi trayectoria como estudiante que respondió a esa lógica en la que no nos preguntamos por qué hay que conocer lo que se supone que tenemos que conocer. Solo nos dicen que lo hagamos y muy pocas veces cuestionamos a quiénes leemos y de quiénes aprendemos, y se vuelven referentes. Para Gayatri Chakravorty Spivak (2017), hacer frente a una condición poscolonial es apropiarse de las historias alternativas. Encuentro más propicio ver, por ejemplo, cómo se negocian las narrativas históricas, ya que, con la distancia pertinente, nos dan una clave en la disyuntiva/conjunción sobre cómo reclamar nuestro derecho al conocimiento y asegurar la base del poder cultural donde nos estamos enunciando.

Podemos situarnos desde la obtención del conocimiento y problematizar las maneras que tenemos de adquirirlo, cuantificarlo y socializarlo. La relación más temprana que poseemos con la ordenación del mundo es en la familia, donde aprendemos a relacionarnos con el mundo. De ahí, pronto pasamos a la escolarización por varios años. 
Es acá donde el rango de enseñanza se hace evidente con la relación de dominio jerárquico que se establece ya desde los primeros momentos. Y es aquí donde se puede identificar, como lo menciona Santoni (2001), el entretejido de los estímulos de un cúmulo de experiencias, en el marco en el que se ha crecido. Estas acompañan la experiencia de vida, en conjunto con la educación formal, no formal e informal, lo cual permite que se torne en enseñanza compuesta.

Trama de concretas reacciones conocidas y desconocidas ante estímulos de enseñanza formal e informal, pero también de experiencia de vida, de continuas modificaciones de mentalidad heredada y formada en edades precedentes en la micro o macro comunidad en la que se ha crecido (Santoni Ruigiu, 2001, p. 27).

En los centros de formación académica artística se evidencia que coexistimos como consecuencia de una colonización, lo que resulta en territorios donde se ejerce una educación basada en competencias. Este modelo de aprendizaje prioriza que las y los estudiantes sean competentes, de modo que las evaluaciones se harán desde el parámetro del dominio de las habilidades que han adquirido. Es así como, de una forma disimulada, se ejerce el sometimiento y la invisibilización de los cuerpos. Asimismo, se perpetúan prácticas a partir de construcciones que siguen respondiendo, de una u otra forma, a un proceso de estructura en la cual estamos sometidos a una lógica neoliberal que valida referentes de carácter universal, y se deha de lado los saberes tradicionales propios del lugar. En una crítica a la escolarización, Iván Illich evidencia a una sociedad de las instituciones que enmarca el progreso tecnológico en los sistemas educativos como centros de adiestramiento.

Muchos estudiantes, en especial los que son pobres, saben intuitivamente qué hacen por ellos las escuelas. Los adiestran a confundir proceso y sustancia. Una vez que estos dos términos se hacen indistintos, se adopta una nueva lógica: cuando más tratamiento haya, tanto mejor serán los resultados. Al alumno se le "escolariza" de ese modo para confundir enseñanza con saber, promoción al curso siguiente con educación, diploma con competencia y fluidez con capacidad para decir algo nuevo. A su imaginación se la "escolariza" para que acepte servicio en vez de valor. Se confunde el tratamiento médico tomándolo por cuidado de la salud, el trabajo social por mejoramiento de la vida comunitaria, la protección policial por tranquilidad, el equilibrio militar por seguridad nacional, la mezquina lucha cotidiana por trabajo productivo. La salud, el saber, la dignidad, la independencia y el quehacer creativo quedan definidos como poco más que el desempeño de las instituciones 
que afirman servir a estos fines, y su mejoramiento se hace dependiente de la asignación de mayores recursos a la administración de hospitales, escuelas y demás organismos correspondientes (Illich, 2011, p. 25).

Dentro de la conformación de la economía, la educación artística parte de presupuestos sobre las conductas creativas y estéticas desde las infancias. Así, dispone de estas actividades en la lógica productivista y las enarbola como mera manualidad, fuera del contexto de la localidad donde habitan las infancias, de forma que resultan receptoras(es) en su desarrollo educativo como espectadoras(es) de objetos estéticos de consumo masivo, en donde no hay cabida para las tradiciones propias que se cargan en el cuerpo. Cabe subrayar la identificación en lo multicultural como una salida existente a la diferencia, propuesta generada en la posmodernidad, que no da cabida más que para controlar, por medio de gavetas, a las diversidades tanto culturales como sexuales y que se complejiza en la condición póstuma (Garcés, 2018, p. 13), pues ya no hay tiempo para el presente que prometía la globalización. Se nos agotaron los recursos y las esperanzas puestas en ciertos modelos que creíamos inamovibles. Ahora, todo es insostenible y con la pandemia es laxo el tiempo que no entendemos. Se nos acabaron las certezas y tampoco se nos permite abarcar la complejidad de la diversidad de cada cultura.

\section{A manera de cierre}

Desde mi experiencia docente en la universidad pública, desde la Licenciatura en Artes Visuales, he impartido materias de índole teórico. Al revisar a las y los autoras(es) esas(os) que se tienen que enseñarse y están en el plan de estudios, me doy cuenta que no es la única vía para armar un panorama amplio del campo de conocimiento del arte occidental. Hay que abrir el abanico de posibilidades a otras formas de producción cultural. En los primeros años como docente, no me cuestioné mucho esto: solo había que comprenderles y tratar de construirlas(os) en el aula entre todas(os).

Con los años y las búsquedas de carácter personal, sumado a estar como tallerista dentro de una institución que trabaja desde la educación artística no formal, surgieron nuevos cuestionamientos que se daban en el calor de la discusión dentro del aula. Al preguntarme el por qué utilizar particularmente a esos autores y autoras, su pertinencia y, sobre todo, su incidencia, y analizar los programas de estudio de las asignaturas que impartía me pareció necesario hacer cruces con autoras(es) contemporáneas(os). No solo eso, había que repensar en esos otras(os) autoras(es) con visión crítica de lo que ya tenemos establecido. 
Un ejemplo de cómo llegar a un mito fundacional y construirlo desde otro lugar fue al toparme con el libro La modernidad de lo barroco (Echeverría, 2011) y pensar en el poder y la responsabilidad de la traducción, ya que no existe una traducción sin una carga política e ideológica. Otro ejemplo de ello fue encontrarme con una conferencia-performance de María Gimeno (s.f.), quien, con su proyecto Queridas viejas, incluye en el libro de Ernest Gombrich los nombres de las artistas mujeres que han sido omitidos. Aquí se devela un problema que ocurre desde que la humanidad cuenta su propia existencia y descarta el papel de las mujeres. Gombrich, sin duda, es un referente para estudiar la historia del arte, esa historia escrita con mayúsculas y que deja de lado los procesos humanos, ya que nos habla de productos terminados y listos para ser analizados por elementos formales que lo conforman.

Así, nos encontramos con la costumbre de ponderar el conocimiento académico, como pasa de forma regular en la Facultad de Artes y Diseño, por medio de esos autores que responden a una cultura universal y que se suponen necesarios y fundamentales para una educación lineal; además que responden a preceptos modernos. Si bien es necesario entenderlos y revisarlos a profundidad para identificar desde qué estructura corresponde el ejercicio de hegemonía en la que participamos de forma indirecta, hace falta y está como urgencia el revalorar los conocimientos de carácter popular y sumarlos como sucede en la Fábrica de Artes Oficios. Asimismo, hay que reconocer la multiplicidad de relatos de forma singular y colectiva, ya que estos influyen en los territorios y los territorios influyen en ellos. 


\section{Referencias}

Acaso, M. (2016). Reduvolution, hacer la revolución en la educación. Madrid, España: Paidós.

Corona Berkin, S. (2019). Producción horizontal del conocimiento. Guadalajara, México: Calas.

Echeverría, B. (2011). La modernidad de lo barroco. Ciudad de México, México: Ediciones Era.

Gómez Grijalva, D. A. (2014). Mi cuerpo es un territorio político. En Espinosa Miñoso, Y., Gómez Correal, D. \& Ochoa Muñoz, K.(eds.) Tejiendo de otro modo: Feminismo, epistemología y apuestas descoloniales en Abya Yala. (pp. 263-277) Colombia: Universidad del Cauca.

Fundación UNAM. (s.f.). Celebra la UNAM 90 años de autonomía. Fundación UNAM. [Página web]. Recuperado de https://www.fundacionunam.org.mx/donde-paso/celebra-unam-90-anos-de-autonomia

Garcés, M. (2018). Nueva ilustración radical. Barcelona, España: Anagrama.

Gimeno, M. (s.f.). Queridas viejas. Perfromances. María Gimero. [Página web]. Recuperado de https://www.mariagimeno.com/QUERIDAS-VIEJAS-PERFROMANCES

Giuliano, F., \& Berisso, D. (2014). Educación y decolonialidad: aprender a desaprender para poder re-aprender. Un diálogo geopolítico-pedagógico con Walter Mignolo. Revista del IICE, 31, 61-71.

Haraway, D. (2020). Manifiesto Cíborg. Madrid, España: Kaotica Libros.

hooks, b. (2003). Teaching Community. A Pedagogy of Hope. New York, USA: Routledge.

hooks, b., Anzaldúa, G., Sandoval, C., \& Brah, A. (2004). Otras inapropiables. Feminismos desde las fronteras. Madrid, España: Traficantes de Sueños.

Illich, I. (2011). La sociedad desescolarizada. Buenos Aires, Argentina: Ediciones Godot.

Jabardo Velasco, M. (2012). Introducción Construyendo puentes: en diálogo desde/con el feminismo negro. Feminismos negro. Una antología. (pp. 27-55). Madrid, España: Traficantes de sueños. 
Contenedor de voces desde la narrativa propia frente

Reflexión

a los discursos dominantes en la educación artística

Rancière, J. (2011). El espectador emancipado. Buenos Aires, Argentina: Manantial.

Rodríguez, S. (1828). Dos extractos de sociedades americanas en 1828. Edición facsimiladocumentada y anotada. (Facsímil digitalizado por el grupo Inventamos o erramos). Ciudad de México, México: Universidad Aautónoma de la Ciudad de México.

Rolnik, S., \& Guattari, F. (2006). Micropolítica. Cartografía del deseo. Madrid, España: Traficantes de Sueños.

Santoni Ruigiu, A. (2001). Escenarios: una aportación dramática a la educación. Ciudad de México, México: Centro de Estudios Sobre la Universidad- Universidad Nacional Autónoma de México/Fondo de Cultura Económica.

Spivak, G. C. (2017). Una educación estética en la era de la globalización. Ciudad de México, México: Universidad Nacional Autónoma de México.

Universidad Nacional Autónoma de México. (s.f.). Programa de Apoyo a Proyectos para Innovar y Mejorar la Educación (PAPIME). Dirección General de Asuntos del Personal Académica. [Página web]. Recuperado de https://dgapa.unam.mx/index.php/fortalecimiento-a-la-docencia/papime

ESCENA. Revista de las artes, 2021, Vol. 81, Núm. 1 (julio-diciembre), pp. 420-434 